\title{
PRODUÇÃO ANIMAL EM PASTAGEM DE MILHETO SOB DIFERENTES NÍVEIS DE NITROGÊNIO ${ }^{1}$
}

\author{
EDUARDO LONDERO MOOJEN ${ }^{2}$, JOÃO RESTLE ${ }^{3}$, GELCI CARLOS LUPATINI ${ }^{4}$ e ADAUTO GOMES DE MORAES ${ }^{5}$
}

\begin{abstract}
RESUMO - O experimento foi conduzido em área da Universidade Federal de Santa Maria, RS, com o objetivo de verificar os efeitos de três níveis de adubação nitrogenada $(0,150$ e $300 \mathrm{~kg} / \mathrm{ha}$ de N) em pastagem de milheto (Pennisetum americanum (L.) Leeke) cv. Comum, sobre a produção animal. Foram utilizados novilhos de corte e avaliados o desempenho por animal, o número de animais.dia/ha e o ganho de peso vivo por área. O sistema de pastejo adotado foi o contínuo, com ajustes de carga para manter uma pressão de pastejo de $10 \%$, caracterizando um resíduo médio de $3.168 \mathrm{~kg}$ de matéria seca/ha. As variáveis dependentes mostraram relação linear positiva com os níveis de adubação nitrogenada, o que denota o alto potencial do milheto.
\end{abstract}

Termos para indexação: adubação nitrogenada, carga animal, desempenho por animal, novilhos, produtividade animal, uréia.

\section{ANIMAL PRODUCTION IN PEARL MILLET PASTURE UNDER DIFFERENT NITROGEN LEVELS}

ABSTRACT - An experiment was conducted at the Universidade Federal de Santa Maria, in Santa Maria, RS, Brazil, with the objective of evaluating the effects of three $\mathrm{N}$ levels $(0,150$ and $300 \mathrm{~kg} / \mathrm{ha})$, in pearl millet (Pennisetum americanum (L.) Leeke) cv. Comum, on animal production. Beef steers were assayed through daily liveweigh gain, gain per hectare and animals.day/hectare. A continuous grazing system was used, with stocking rate adjustments to maintain a $10 \%$ grazing pressure that characterized a medium residue of $3,168 \mathrm{~kg}$ of DM/ha. The dependent variables showed a positive linear relationship with the $\mathrm{N}$ levels, denoting the high animal production potential of pearl millet.

Index terms: nitrogen fertilization, carrying capacity, animal performance, beef steers, animal productivity, urea.

\section{INTRODUÇ̃̃O}

A pesquisa em forragicultura no âmbito da avaliação da produção animal em pastagens busca alternativas de espécies forrageiras para os diversos sistemas de produção pecuários. Neste contexto, $\mathrm{o}$ milheto (Pennisetum americanum) é, atualmente, uma forrageira cultivada de grande importância. Seu cultivo é realizado principalmente em sistemas de

${ }^{1}$ Aceito para publicação em 26 de fevereiro de 1999.

2 Eng. Agr., Dr., Prof. Titular, Dep. de Zootecnia, Universidade Federal de Santa Maria (UFSM), CEP 97119-900 Santa Maria, RS. E-mail: moojenel@creta.ccr.ufsm.br

${ }^{3}$ Eng. Agr., Ph.D., Prof. Titular, Dep. de Zootecnia, UFSM Bolsista do CNPq.

${ }^{4}$ Eng. Agr., M.Sc., Dep. de Zootecnia, UFSM

5 Eng. Agr., Dep. de Zootecnia, UFSM. produção animal mais avançados. Sua característica principal é um alto potencial de produção de forragem com alta qualidade num período reduzido, no qual pode suportar pesadas cargas.

O N é o fator que mais limita a produção de forragem em ecossistemas de pastagens do mundo. Dentro de certos limites, ao ser adicionado ao solo provoca aumentos no rendimento de matéria seca e teor de proteína bruta (PB) na cultura de milheto (Hart \& Burton, 1965).

Com a cultivar Gahi-1 de milheto, Hart \& Burton (1965) obtiveram uma resposta linear em produção de matéria seca para níveis de 0 a $600 \mathrm{~kg} / \mathrm{ha}$ de N.

Medeiros (1972), avaliando milheto comum no Rio Grande do Sul, com cortes simulando pastejo controlado, e sob o efeito de níveis de $\mathrm{N}(0,100$, 200 e $300 \mathrm{~kg} / \mathrm{ha}$ ), concluiu que o $\mathrm{N}$ aumentou o 
rendimento total de matéria seca (MS), bem como o teor e a produção de PB. Quantificou produções de MS de 7,9, 13,2, 17,7 e 18,2 t/ha, para os níveis testados, respectivamente. A relação linear $(\mathrm{P}<0,047)$ expressou a relação entre MS e N.

Segundo Fribourg (1985), respostas lineares de gramíneas anuais de verão à fertilização nitrogenada acima de $200 \mathrm{~kg} / \mathrm{ha}$, têm sido obtidas. Para altos níveis de adubação, o parcelamento é essencial para o crescimento uniforme e o equilíbrio nutricional das plantas. No verão, em climas úmidos, de 15 a 20 t/ha de MS, podem ser conseguidas com aplicações de $400 \mathrm{~kg} / \mathrm{ha} \mathrm{de} \mathrm{N}$, ou mais.

Teores adequados de $\mathrm{K}$ e $\mathrm{P}$, especialmente o de $\mathrm{K}$, aumentam a eficiência das plantas em utilizar altas doses de $\mathrm{N}$ e transformá-lo em proteína (Lopes \& Guilherme, 1989).

No Rio Grande do Sul, Cóser \& Maraschin (1983) observaram, em pastagem de milheto, um ganho médio diário (GMD) de $0,78 \mathrm{~kg}$ e um ganho de peso vivo (PV) de $479 \mathrm{~kg} / \mathrm{ha}$, num período de 125 dias, com uma lotação média de 8,12 novilhas de $200 \mathrm{~kg}$, com um resíduo superior a $1.200 \mathrm{~kg} / \mathrm{ha}$ de MS. Maraschin (1979) aconselha que seja mantido um resíduo em torno de $2.000 \mathrm{~kg} /$ ha de MS (20 a $30 \mathrm{~cm}$ de altura).

O milheto é capaz de permitir um ganho de peso vivo de $1,0 \mathrm{~kg} / \mathrm{animal} / \mathrm{dia}$ e $400 \mathrm{~kg} / \mathrm{ha}$ de peso vivo, durante 90 dias (McCartor \& Rouquette Junior, 1977). Os autores informam que o ganho de peso vivo depende da forragem disponível, pressão de pastejo e qualidade, e que tanto a pressão de pastejo muito alta como a muito baixa reduzem o ganho.

Dunuvin (1970) obteve um ganho de $401 \mathrm{~kg} / \mathrm{ha}$ de PV com a cultivar Gahi-1. McCartor \& Rouquette Junior (1977) registraram um ganho máximo de $473 \mathrm{~kg} / \mathrm{ha}$ de PV. Moraes \& Maraschin (1988), trabalhando com pressões de pastejo de $4,6,8$ e $10 \%$, obtiveram um GMD de 0,50 a $1,21 \mathrm{~kg}$ e 0,50 a $1,24 \mathrm{~kg}$, para os grupos genéticos Charolês e Zebu, respectivamente, com um ganho por hectare que variou de 259 a $572 \mathrm{~kg}$ de PV. Os mesmos autores citam que com o manejo adequado do milheto, pode-se obter até $7 \mathrm{~kg} / \mathrm{ha} /$ dia de ganho de PV. Concluíram que diferentes pressões de pastejo modificam bruscamente a condição da pastagem do milheto, cujos resíduos de MS vão definir distintos desempenhos tanto por animal como por área.

O presente trabalho objetiva determinar os efeitos de doses crescentes de $\mathrm{N}$ em pastagem de milheto sobre a produção animal.

\section{MATERIAL E MÉTODOS}

O experimento foi conduzido em área do Departamento de Zootecnia da Universidade Federal da Santa Maria, RS. Santa Maria situa-se na região fisiográfica da Depressão Central, com altitude média de $95 \mathrm{~m}, 29^{\circ} 42^{\prime}$ de latitude Sul e $53^{\circ} 42^{\prime}$ de longitude Oeste.

O clima da região, segundo a classificação de Köppen, é Cfa (Subtropical úmido), com precipitação média anual de $1.769 \mathrm{~mm}$; temperatura média anual de $19,2^{\circ} \mathrm{C}$, insolação de 2.212 horas anuais e umidade relativa do ar de $82 \%$ (Moreno, 1961).

O solo onde foi conduzido o experimento classifica-se como Podzólico Vermelho-Amarelo distrófico, pertencendo à unidade de mapeamento São Pedro.

Os tratamentos constaram de três níveis de adubação nitrogenada em cobertura em pastagem de milheto cv. Comum: $\mathrm{N}_{0}=0 \mathrm{~kg} /$ ha de $\mathrm{N}$ (testemunha); $\mathrm{N}_{1}=150 \mathrm{~kg} / \mathrm{ha} \mathrm{de}$ $\mathrm{Ne} \mathrm{N}_{2}=300 \mathrm{~kg} /$ ha de $\mathrm{N}$. O adubo nitrogenado utilizado foi a uréia. A adubação de cobertura foi fracionada em três aplicações a lanço em 29/12/91,29/01 e 26/2/92 e aplicada a lanço.

O delineamento experimental adotado foi em blocos completos casualizados, com três tratamentos e três repetições.

As unidades experimentais possuíam áreas de $0,904 \mathrm{a}$ 2,115 ha, totalizando uma área de 11 ha; quanto maior a dosagem de $\mathrm{N}$, menor a área das unidades experimentais com o propósito de manter, no mínimo, três animais por unidade experimental. Utilizou-se também uma área adjacente com, aproximadamente, 8 ha, para manter os animais regulares (put-and-take)

O preparo do solo constou de uma escarificação, seguida de aração com arado gradeador e duas gradagens leves com uma grade de dentes. Foi feita uma terceira gradagem leve, imediatamente antes da semeadura.

A adubação básica (P e K) seguiu a recomendação de Siqueira et al. (1987) sendo feita individualmente por unidade experimental.

Foi utilizada uma densidade de semeadura de $22 \mathrm{~kg} / \mathrm{ha}$ de sementes com valor cultural de $75 \%$, o que correspondeu a uma densidade de $16,5 \mathrm{~kg} / \mathrm{ha}$ com valor cultural de $100 \%$. A semeadura foi realizada em 7/12/91, com uma semeadeira Fundiferro, em linhas com espaços de $0,40 \mathrm{~cm}$, com posterior passagem de um rolo compactador. Da semeadura ao 
início da utilização, transcorreram 32 dias. O período de utilização sob pastejo foi de 8/1/92 a 3/4/92 (86 dias).

Foram usados animais testers e put-and-take (Mott \& Lucas, 1952), pertencentes aos grupos genéticos Charolês, Nelore e seus cruzamentos. Os animais testers foram novilhos com idade entre 13 e 14 meses, com peso médio de $260 \mathrm{~kg}$ (peso médio inicial e final de cerca de, respectivamente, $230 \mathrm{~kg}$ e $290 \mathrm{~kg}$ ). Os animais de todos os tratamentos tiveram acesso à mistura mineral. A vacinação antiaftosa seguiu o calendário da Inspetoria Veterinária da Secretaria da Agricultura e Abastecimento do Rio Grande do Sul. O controle do carrapato foi realizado quando necessário, e a vermifugação, feita sistematicamente.

O ajuste da carga animal (CA) foi realizado a cada 28 dias, para uma pressão de pastejo (PP) de 10\% (10 kg de $\mathrm{MS} / 100 \mathrm{~kg}$ de PV/dia), deixando-se um resíduo de cerca de $3.000 \mathrm{~kg} / \mathrm{ha}$ de MS e acrescentando-se a este o crescimento previsto para o período, o que representou a forragem disponível

Os animais foram pesados com jejum prévio de 14 horas no início e final do experimento, e nas pesagens intermediárias foi adotado um jejum de seis horas. Por diferença de peso foi quantificado o ganho médio diário (GMD) dos novilhos no período total.

A variável animais.dia/ha $(\mathrm{AD} / \mathrm{ha})$ foi calculada pelo total de novilhos por tratamento, somando-se todos os períodos, e a carga animal (CA) média expressa em $\mathrm{kg} / \mathrm{ha}$ de peso vivo foi calculada pela média de peso vivo por tratamento, em cada período.

Os ganhos de peso vivo (GPV) por área foram calculados multiplicando-se animais.dia/ha pelo ganho médio diário.

Foram realizadas análises de regressão das variáveis dependentes, tendo em vista os níveis de $\mathrm{N}$ aplicados. As análises foram feitas utilizando-se o sistema computacional SAS (1985).

\section{RESULTADOS E DISCUSSÃO}

O GMD apresentou uma relação linear positiva $(\mathrm{P}<0,0925)$ com os níveis de adubação nitrogenada (Fig. 1). Os ganhos variaram de $0,553 \mathrm{~kg}$ a $0,764 \mathrm{~kg}$.

De acordo com a literatura, pode-se, ou não, esperar mudanças no desempenho por animal em face da fertilização nitrogenada (Dougherty \& Rhykerd, 1985; Favoretto et al., 1985).

O número de animais.dia/ha aumentou linearmente $(\mathrm{P}<0,0003)$ com o aumento dos níveis de $\mathrm{N}$ (Fig. 2). Pela regressão, pode-se observar que o número de animais.dia/ha praticamente dobrou quando o nível de $\mathrm{N}$ passou de zero para $300 \mathrm{~kg} / \mathrm{ha}$, pois para um mesmo nível de oferta de forragem nos tratamentos, níveis mais altos de $\mathrm{N}$ resultaram em maior produção de forragem, estes, como conseqüência, suportaram maior carga animal, o que já era esperado.

A relação entre a carga animal média expressa em peso vivo/ha e os níveis de $\mathrm{N}$ é vista na Fig. 3. Observa-se que com o aumento dos níveis de $\mathrm{N}$ houve

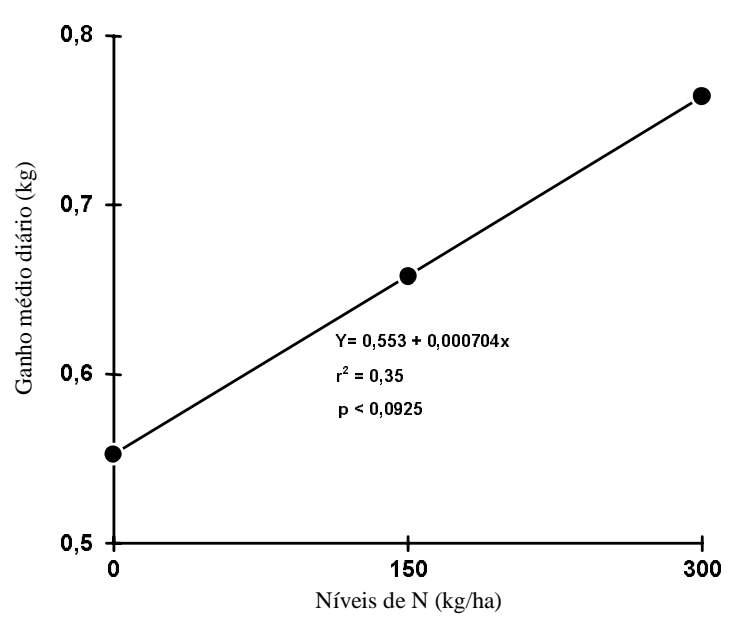

FIG. 1. Ganho médio diário obtido em pastagens de milheto em função dos níveis de nitrogênio aplicados.

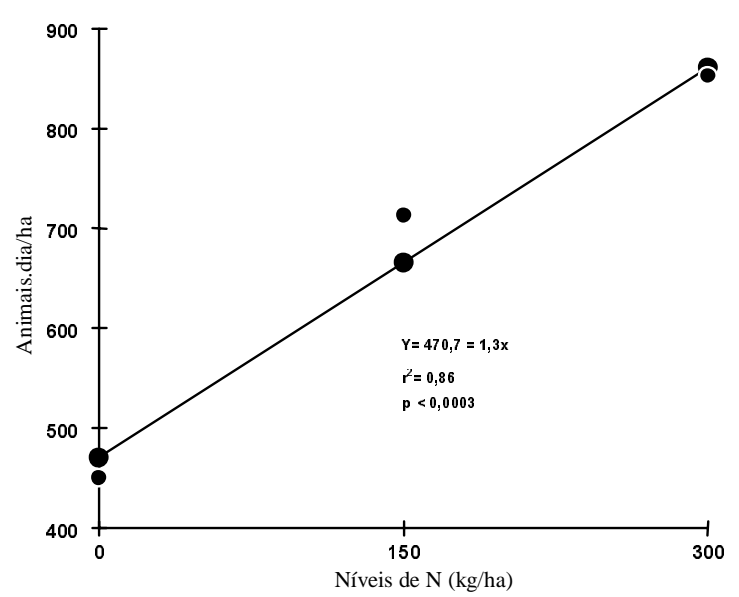

FIG. 2. Animais.dia/ha em pastagens de milheto em função dos níveis de nitrogênio aplicados. 
um aumento linear na carga suportada. A carga praticamente dobrou no nível mais alto de $\mathrm{N}$ em relação ao mais baixo. A variação de carga foi de 1.581 a $2.871 \mathrm{~kg} / \mathrm{ha}$ de peso vivo.

Na Fig. 4, observa-se que, à medida que aumentou o nível de $\mathrm{N}$, houve um aumento linear $(\mathrm{P}<0,0005)$ no GPV/ha, que variou de 245 a $665 \mathrm{~kg} / \mathrm{ha}$, entre os níveis zero e $300 \mathrm{~kg} / \mathrm{ha}$. Os

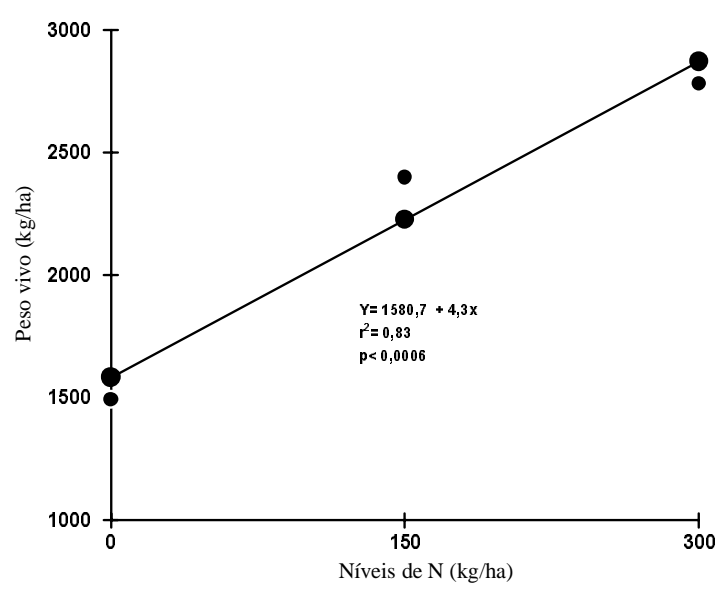

FIG. 3. Carga animal média expressa em peso vivo (kg/ha) em pastagens de milheto em função dos níveis de nitrogênio aplicados.

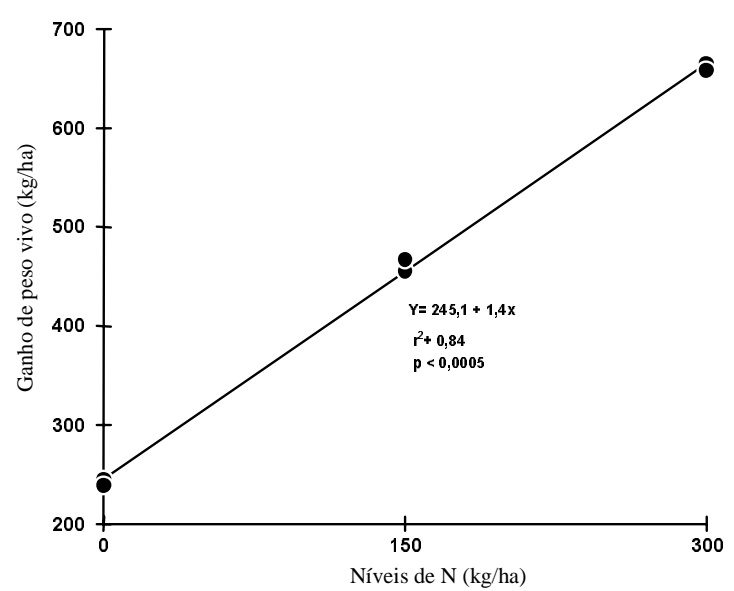

FIG. 4. Ganho de peso vivo por área dos novilhos em pastagens de milheto em função dos níveis de nitrogênio aplicados. ganhos obtidos neste trabalho superam os de outros autores com a mesma espécie no Rio Grande do Sul. Isto é explicado pelos maiores níveis de $\mathrm{N}$ utilizados neste trabalho, em relação aos de Cóser \& Maraschin (1983) e Moraes (1984). Deve-se mencionar que o nível de oferta de forragem neste trabalho foi igual ao do melhor tratamento relatado por Moraes (1984).

\section{CONCLUSÕES}

1. Há resposta linear do ganho médio diário, do número de animais.dia/ha e do ganho de peso vivo por área a doses de nitrogênio.

2. Maiores doses de nitrogênio permitem aumentar a carga animal em pastagens de milheto.

\section{REFERÊNCIAS}

CÓSER, A.C.; MARASCHIN, G.E. Desempenho animal em pastagens de milheto comum e sorgo. Pesquisa Agropecuária Brasileira, Brasília, v.18, n.4, p.421-426, abr. 1983.

DOUGHERTY, C.T.; RHYKERD, C.L. The role of nitrogen in forage-animal production. In: HEATH, M.E.; BARNES, R.F.; METCALFE, D.S. (Eds.). Forages: the science of grassland agriculture. 4.ed. Ames : Iowa State University, 1985. p.318-325.

DUNUVIN, L.S. Gahi pearl millet and two sorghum $\mathrm{x}$ sudangrass hybrid as pasture for yearling beef cattle. Agronomy Journal, Madison, v.62, n.3, p.375-377, 1970.

FAVORETTO, V.; REIS, R.A.; VIEIRA, P.F.; MALHEIROS, E.B. Efeito da adubação nitrogenada ou de leguminosas no ganho de peso vivo de bovinos em pastagens de capim colonião. Pesquisa Agropecuária Brasileira, Brasília, v.20, n.4, p.475-482, 1985.

FRIBOURG, H.A. Summer anual grasses. In: HEATH, M.E.; BARNES, R.F.; METCALFE, D.S. (Eds.). Forages: the science of grassland agriculture. 4.ed. Ames : Iowa State University, 1985. 643p.

HART, R.H.; BURTON, G.W. Effect of row spacing seeding rate and nitrogen fertilization on forage yield and quality of Gahi-1 pearl millet. Agronomy Journal, Madison, v.57, n.4, p.376-378, 1965. 
LOPES, A.S.; GUILHERME, R. Uso eficiente de fertilizantes. In: SIMPÓSIO AVANÇADO DE SOLOS E NUTRIÇÃO DE PLANTAS, 2., 1989, Piracicaba Anais. São Paulo : ESALQ, 1989. p.1-58

McCARTOR, M.M.; ROUQUETTE JUNIOR, F.M Grazing pressures and animal performance from pearl millet. Agronomy Journal, Madison, v.69, n.6, p.983-987, 1977.

MARASCHIN, G.E. Potencial produtivo de gramíneas forrageiras de verão no sul do Brasil. Lavoura Arrozeira, Porto Alegre, v.32, n.315, p.18-24, ago. 1979.

MEDEIROS, R.B. Efeito do nitrogênio e população de plantas sobre o rendimento de matéria seca, teor e produção de proteína bruta de sorgo e milheto forrageiros. Porto Alegre : UFRGS, 1972. 96p. Dissertação de Mestrado.

MORAES, A. de. Pressões de pastejo e produção animal em milheto (Pennisetum americanum (L.) Leeke). Porto Alegre : UFRGS, 1984. 104p. Dissertação de Mestrado
MORAES,A. de; MARASCHIN, G.E. Pressões de pastejo e produção animal em milheto $\mathrm{cv}$. comum. Pesquisa Agropecuária Brasileira, Brasília, v.23, n.2, p.197-205, 1988.

MORENo, J.A. Clima do Rio Grande do Sul. Porto Alegre : Secretaria da Agricultura, 1961.41p.

MOTT, G.O.; LUCAS, H.L. The design, conduct and interpretation of grazing trials on cultivated and improved pastures. In: INTERNATIONAL GRASSLAND CONGRESS, 6., 1952, State College. Proceedings. State College, Pensylvania : State College Press, 1952. p.1380-1385.

SAS INSTITUTE. SAS user's guide. 5.ed. Cary, NC, 1985. 429p.

SIQUEIRA, O.J.F.; SCHERER, E.E.; TASSINARI, G.; ANGHINONI, I.; PATELLA, J.F.; TEDESCO, M.J.; MILAN, P.A.; ERNANI, P.R.; WIETHOLTER, $\mathrm{S}$. Recomendações de adubação e calagem para os estados de Rio Grande do Sul e Santa Catarina Passo Fundo : Embrapa-CNPT, 1987. 100p. 